\title{
Exploitation of Heterosis for Yield and its Components in Rice
}

\author{
R. Krishna Naik*, P. Ramesh Babu, J. Dayal Prasad Babu, \\ Y. Ashoka Rani and V. Srinivasa Rao
}

Department of Genetic \& Plant Breeding, Agricultural College, Bapatla 522 101, A.P., India

*Corresponding author

\begin{abstract}
A B S T R A C T
Keywords

Rice (Oryza sativa L.), Heterosis, Yield,

Components

Article Info

Accepted:

12 August 2018

Available Online:

10 September 2018

Present study was conducted involving 54 rice hybrids to study standard heterosis expression for yield and some yield components characters viz., days to 50 per cent flowering, days to maturity, plant height, panicle length, productive tillers per plant, filled grains panicle $^{-1}$, total number of grains panicle ${ }^{-1}$, spikelet fertility, test weight and grain yield plant ${ }^{-1}$. Among the heterotic crosses IR-58025A x WGL 3962 and IR-68897A x BPT 5204 showed significant negative standard heterosis for days to $50 \%$ flowering, days to maturity and plant height, so that this will be effective for developing short duration, semidwarf rice hybrids and also showed significant positive standard heterosis over KRH2 for number of filed grains per panicle, total number of grains, test weight and grain yield per plant. So these promising cross combinations could be further used in rice breeding programme for developing high yielding rice hybrids
\end{abstract}

\section{Introduction}

Rice (Oryza sativa L., $2 \mathrm{n}=24$ ) is the most important food crop in the world (Asia, Latin America, parts of Africa and the Middle East), which accounts for more than $21 \%$ of the calorific needs of the world's population and up to $76 \%$ of the calorific intake of the population of South East Asia.. About 90\% of the world's rice is grown and consumed in Asia.

According to the projections made by the Population Foundation of India, the country's population will be 1546 by the end of 2030 and 1824 million by the end of 2050 from the present population of 1234 million. It is estimated that the demand for rice will be
121.2 million tonnes by the year 2030 and 137.3 million tonnes by the year 2050. (CRRI -VISION 2050).

India is the second largest country after China, which has implemented hybrid rice technology on a commercial scale. Government of India launched a mission mode project on hybrid rice during 1989 and Andhra Pradesh was the first state in India to release two hybrids, APHR-1 and APHR-2 during the year 1994 from Regional Agricultural Research station, APRRI, Maruteru for commercial cultivation. As a result of concerted research and development efforts for more than two decades by the Indian scientists, a total of 93 hybrids have been released so far from 1994 to 2017 for 
commercial cultivation including 55 and 38 hybrids from private and public sector respectively. The area under hybrid rice reached one million hectare in 2006, from the initial level of 10,000 hectares in 1995 and exceeded 2.5 million hectares during 2014, which was about $5.6 \%$ of the total rice area in the country and it was estimated that around more than three million hectares is under hybrid rice cultivation in India in 2016 which was around $7 \%$ of the total rice cropped area in India. Rice yields increased by about $30 \%$ due to the development of semi dwarf varieties, and an additional $15-20 \%$ was achieved through the use of heterosis. Hybrid rice is a proven and economically viable option to boost rice production. Hence, the present study was undertaken to assess the extent of heterosis and to identify best cross combinations on the basis of grain yield traits for future utilization in breeding programmes

\section{Materials and Methods}

The present investigation was carried out at Agricultural Research Station, Nellore during rabi 2015-16 with the objective of estimate genetic variability among the parents and rice hybrids. The experimental materials for the present study consisted of 54 hybrids produced by $\mathrm{L}$ x $\mathrm{T}$ mating design. The experimental material was planted in four rows with a row length of $4.5 \mathrm{~m}$ and two replications in a Randomized Block Design (RBD). Twenty five days old seedlings were transplanted with a spacing of $20 \mathrm{~cm}$ between rows and $15 \mathrm{~cm}$ between plants under recommended dose of fertilizers i.e., $120 \mathrm{~N}$ : 40 $\mathrm{P}_{2} \mathrm{O} 5: 40 \mathrm{~K}_{2} \mathrm{O}$ per hectare along with necessary plant protection measures to raise a good crop. Observations were recorded for ten characters on ten randomly selected plants of each replication and the mean data was subjected to statistical analysis. The estimates of standard heterosis over high yielding and popular hybrid check KRH-2 was estimated for ten characters viz., days to 50 per cent flowering, days to maturity, plant height, panicle length, productive tillers per plant, filled grains panicle $^{-1}$, total number of grains panicle ${ }^{-1}$, spikelet fertility, test weight and grain yield plant $^{-1}$. The standard heterosis was estimated as suggested by Liang et al., (1971).

\section{Results and Discussion}

The magnitude of heterosis for yield and yield components traits depends to a large extent on genetic variation, genetic base and adaptability of parents. The hybrids showing high heterosis have good chances to identify desirable lines in succeeding generations as compared to hybrids having low heterotic effects. The estimates of standard heterosis for yield and yield attributing traits were showed that considerable amount of heterosis existed both in positive and negative directions for all the traits (Table 1).

\section{Days to $50 \%$ flowering}

Early flowering hybrids are desirable as they produce more yields per day and fit well in multiple cropping system. Hence, negative heterosis is desirable for the trait as it indicates the earliness of the hybrids. In the present study standard heterosis ranged from -15.51 (IR-68888A x JGL 11118) to 8.56 per cent (IR-58025A x RNR 2458) and twenty five hybrids expressed significant negative and desirable heterosis over check KRH-2.

\section{Days to maturity}

Days to maturity indicates the duration of a genotype, early maturity genotypes reduces the duration of the crop. Hence, it is desirable to choose the genotypes with negative and significant heterosis for this trait. In the present study, standard heterosis for days to maturity was ranged from -9.60 (IR-68888A x JGL 11118) to 8.00 (IR-58025A x NLR 40024 
\& IR-58025A x NLR 3041) and twenty hybrids expressed significant and desirable heterosis over check KRH-2.

\section{Plant height (cm)}

Significant increase in rice yield has been achieved with the development of semi dwarf cultivars characterized by lodging resistance, nitrogen responsiveness and erect leaves. Infact, the quantum jump in grain yield was achieved with intensive use of tropical semi dwarf rice varieties. Negative heterosis was desirable for plant height for breeding short statured hybrids. The standard heterosis was ranged from -36.90 (IR-68888A x NLR 40024) to -11.17 per cent (IR-58025A x PS 4) over check KRH-2. Out of fifty four hybrids evaluated, all the hybrids were exhibited negative heterosis indicated that all these hybrids were dwarf in nature.

\section{Number of productive tillers plant ${ }^{-1}$}

Higher number of productive tillers per plant is one of the important components of yield thus the hybrids with positive heterosis were desirable. In the present study, for the character productive tillers per plant positive and significant heterosis was observed for two hybrids and the heterosis was ranged from 39.55 (IR-58025A x BPT 5204) to 18.59 per cent (IR-58025A x NLR 3010).

\section{Panicle length $(\mathrm{cm})$}

Longer panicle is generally associated with more number of spikelets and this is one of the attributes for higher grain yield in rice hybrids and positive heterosis is desirable for this trait. In the present study, for the character panicle length none of the hybrids recorded significant positive standard heterosis and the was from 34.02 (IR-58025A x NLR 40024 \& IR58025A $x$ ADT 37) to -4.98 (IR-68897A $x$ PS 4 \& IR-68897A x WGL 32100).

\section{Number of filled grains panicle ${ }^{-1}$}

Filled grains per panicle is one of the most important yield attributing trait and probably this trait will be helpful in breaking the yield ceiling, hence significant positive heterosis is desirable. For filled grains per panicle, twenty six hybrids were excelled positive standard heterosis over check KRH-2 and it ranged from - 63.51 (IR-68897A x Sumati) to 59.92 per cent (IR-68897A x MTU1001).

\section{Total number of grains panicle ${ }^{-1}$}

For this characters total number of grains per panicle, the standard heterosis ranged from 58.04 (IR-68897A x Sumati) to 68.44 (IR68897A x NLR 3041) and twenty five hybrids were exhibited significant positive heterosis over check KRH-2.

\section{Spikelet fertility (\%)}

The extent of spikelet fertility is an important character which directly influences the ultimate grain yield. Positive heterosis for spikelet fertility is desirable. Out of fifty four hybrids, one hybrid IR 68888 A x Sumati (10.34) was recorded significant positive heterosis over check KRH-2.

The range of heterosis was from -23.31 (IR68897A x ADT 37) to 10.34 (IR-68888A x Sumati). Low grain yields in rice hybrids are attributed mainly to the high sterility percentage. The extent of spikelet fertility directly influences the ultimate product (grain yield).

\section{Test weight $(g)$}

The fine grain types are generally associated with low test weight for which negative heterosis may be considered and whereas, the positive heterosis is associated with bold grain with more test weight. 
Table.1 Estimates of Standard heterosis for yield and yield components in rice (Oryza sativa L.)

\begin{tabular}{|c|c|c|c|c|c|c|c|c|c|c|c|}
\hline $\begin{array}{c}\text { S. } \\
\text { No. }\end{array}$ & Cross & $\begin{array}{l}\text { Days to } \\
50 \\
\text { flowering }\end{array}$ & $\begin{array}{l}\text { Days to } \\
\text { maturity }\end{array}$ & $\begin{array}{c}\text { Plant } \\
\text { height } \\
(\mathrm{cm})\end{array}$ & $\begin{array}{c}\text { No. of } \\
\text { productive } \\
\text { tillers } \\
\text { plant }^{-1}\end{array}$ & $\begin{array}{l}\text { Panicle } \\
\text { length } \\
(\mathrm{cm})\end{array}$ & $\begin{array}{l}\text { No. of } \\
\text { filled } \\
\text { grains } \\
\text { panicle-1 }\end{array}$ & $\begin{array}{l}\text { Total no. } \\
\text { of grains } \\
\text { panicle- }^{1}\end{array}$ & $\begin{array}{l}\text { Spikelet } \\
\text { fertility } \\
\text { (g) }\end{array}$ & $\begin{array}{c}\text { Test } \\
\text { weight } \\
(\mathrm{g})\end{array}$ & $\begin{array}{l}\text { Grain } \\
\text { yield } \\
\text { plant-1 } \\
\text { (g) }\end{array}$ \\
\hline 1 & IR-58025A x NLR 145 & -0.53 & -0.40 & $-25.45 * *$ & $-21.58 * *$ & $-14.52 * *$ & 11.06 & 10.40 & 0.47 & $-11.86 * *$ & $-31.51 * *$ \\
\hline 2 & IR-58025A x NLR 34449 & $6.42 * *$ & $5.60 * *$ & $-30.75 * *$ & -3.43 & $-18.26 * *$ & $45.13 * *$ & $46.17 * *$ & -0.96 & $-14.57 * *$ & $26.08^{*}$ \\
\hline 3 & IR-58025A x NLR 40024 & $2.14^{*}$ & $8.00 * *$ & $-31.50 * *$ & $-34.77 * *$ & $-34.02 * *$ & -3.22 & -6.00 & 3.02 & -3.78 & $-40.75^{* *}$ \\
\hline 4 & IR-58025A x NLR 3041 & $8.56^{* *}$ & $8.00 * *$ & $-31.07 * *$ & -9.01 & $-25.31 * *$ & $36.56 * *$ & $37.64 * *$ & -0.56 & $-33.11 * *$ & -18.34 \\
\hline 5 & IR-58025A x NLR 3010 & $-5.88 * *$ & $-4.00 * *$ & $-20.89 * *$ & $18.59 * *$ & $-9.13 *$ & $-40.70 * *$ & $-42.55^{* *}$ & 3.49 & $-13.60 * *$ & $-41.13 * *$ \\
\hline 6 & IR-58025A x NLR 3083 & $-2.67 *$ & $-2.40 *$ & $-23.44 * *$ & -7.27 & $-20.75 * *$ & $57.43 * *$ & $62.80 * *$ & -3.43 & $-20.18 * *$ & $22.59 *$ \\
\hline 7 & IR-58025A x ADT 37 & $-9.09 * *$ & $-6.40 * *$ & $-23.65 * *$ & -9.94 & $-34.02 * *$ & 9.26 & 2.16 & 6.69 & 0.39 & -4.99 \\
\hline 8 & IR-58025A x JGL 11118 & 0.53 & 0.40 & $-30.54 * *$ & 3.74 & $-26.97 * *$ & $-20.39 * *$ & -13.58 & -8.04 & $-19.51 * *$ & $-30.05 * *$ \\
\hline 9 & IR-58025A x MTU 1010 & $-4.28 * *$ & -0.80 & $-30.43 * *$ & $-17.97 *$ & $-16.60 * *$ & 9.19 & 17.35 & -7.13 & $-7.60 * *$ & $-25.06^{*}$ \\
\hline 10 & IR-58025A x MTU1001 & 1.07 & -0.40 & $-31.60 * *$ & -6.20 & $-23.65 * *$ & $48.45 * *$ & $45.17 * *$ & 2.23 & $-17.04 * *$ & 18.03 \\
\hline 11 & IR-58025A x RNR 2458 & $8.56^{* *}$ & $7.20 * *$ & $-25.77 * *$ & $-14.36^{*}$ & $-17.43 * *$ & $36.42 * *$ & $55.11 * *$ & $-12.10^{*}$ & $-17.04 * *$ & -2.58 \\
\hline 12 & IR-58025A x PS 4 & 1.07 & 0.40 & $-11.77 * *$ & $-24.92 * *$ & $-17.01 * *$ & -2.00 & 15.60 & $-15.24 * *$ & $-11.67 * *$ & $-48.21 * *$ \\
\hline 13 & IR-58025A x PS 5 & $-4.28 * *$ & $-2.80 *$ & $-19.09 * *$ & -4.24 & $-12.03 * *$ & $-49.76 * *$ & $-45.21 * *$ & -8.24 & $-9.39 * *$ & $-70.96 * *$ \\
\hline 14 & IR-58025A x BPT 5204 & $3.21 * *$ & 3.20 & $-31.81 * *$ & $-39.55^{* *}$ & $-20.33 * *$ & 7.05 & 16.23 & -8.13 & $-12.20 * *$ & $-45.58 * *$ \\
\hline 15 & IR-58025A x Sumati & -1.07 & -0.80 & $-13.36 * *$ & -6.38 & $-8.71 *$ & -3.18 & 0.88 & -4.06 & $-21.01 * *$ & $-28.40 *$ \\
\hline 16 & IR-58025A x WGL 32100 & $3.21 * *$ & $3.20 * *$ & $-18.66 * *$ & -9.18 & $-17.84 * *$ & $24.95 * *$ & $24.62 *$ & 1.08 & $-20.76 * *$ & -16.02 \\
\hline 17 & IR-58025A x WGL 283 & $-3.21 * *$ & $-2.80 *$ & $-23.22 * *$ & $-23.99 * *$ & $-16.18 * *$ & -1.87 & 15.84 & $-14.91 * *$ & $-5.03 *$ & $-38.53 * *$ \\
\hline 18 & IR-58025A x WGL 3962 & $-8.56^{* *}$ & $-6.80 * *$ & $-23.86 * *$ & -10.88 & $-17.84 * *$ & $29.58 * *$ & $28.19 * *$ & 0.96 & $11.28 * *$ & $33.48 * *$ \\
\hline 19 & IR-68888A x NLR 145 & $-3.74 * *$ & $-2.80 *$ & $-24.39 * *$ & -7.76 & $-18.67 * *$ & $26.26 * *$ & $31.03 * *$ & -3.65 & $-12.10 * *$ & 3.56 \\
\hline 20 & IR-68888A x NLR 34449 & $-4.28 * *$ & $-2.40^{*}$ & $-29.06 * *$ & -4.06 & $-22.41 * *$ & -1.73 & 7.57 & -8.79 & $-18.97 * *$ & $-30.87 * *$ \\
\hline 21 & IR-68888A x NLR 40024 & 1.60 & $2.40 *$ & $-36.90 * *$ & -12.88 & $-19.92 * *$ & 9.95 & 15.49 & -4.81 & $-10.45^{* *}$ & -18.21 \\
\hline
\end{tabular}


Table.1 Cond...

\begin{tabular}{|c|c|c|c|c|c|c|c|c|c|c|c|}
\hline $\begin{array}{l}\text { S. } \\
\text { No. }\end{array}$ & Cross & $\begin{array}{l}\text { Days to } \\
50 \\
\text { flowering }\end{array}$ & $\begin{array}{l}\text { Days to } \\
\text { maturity }\end{array}$ & $\begin{array}{l}\text { Plant } \\
\text { height } \\
(\mathrm{cm})\end{array}$ & $\begin{array}{c}\text { No. of } \\
\text { productive } \\
\text { tillers }^{-1} \\
\text { plant }^{-1}\end{array}$ & $\begin{array}{l}\text { Panicle } \\
\text { length } \\
\text { (cm) }\end{array}$ & $\begin{array}{l}\text { No. of } \\
\text { filled } \\
\text { grains } \\
\text { panicle- }{ }^{1}\end{array}$ & $\begin{array}{l}\text { Total no. } \\
\text { of grains } \\
\text { panicle- }^{1}\end{array}$ & $\begin{array}{l}\text { Spikelet } \\
\text { fertility } \\
\text { (g) }\end{array}$ & $\begin{array}{c}\text { Test } \\
\text { weight } \\
\text { (g) }\end{array}$ & $\begin{array}{l}\text { Grain } \\
\text { yield } \\
\text { plant- } \\
\text { (g) }\end{array}$ \\
\hline 22 & IR-68888A x NLR 3041 & $-8.56^{* * *}$ & $-6.00 * *$ & $-31.60 * *$ & -4.28 & $-25.73 * *$ & $27.16^{* *}$ & & -1.81 & $-18.64 * *$ & 0.44 \\
\hline 23 & IR-68888A x NLR 3010 & $-3.21 * *$ & -2.00 & $-24.39 * *$ & -7.00 & $-17.84 * *$ & $30.41 * *$ & $31.53 * *$ & -1.00 & $-9.78 * *$ & 14.74 \\
\hline 24 & IR-68888A x NLR 3083 & $2.67 *$ & $2.80^{*}$ & $-29.48 * *$ & 10.57 & $-25.73 * *$ & $-34.49 * *$ & $-34.88 * *$ & 0.91 & $-17.23 * *$ & $-43.84 * *$ \\
\hline 25 & IR-68888A x ADT 37 & $2.14 *$ & $2.40 *$ & $-16.86^{* *}$ & $-22.56 * *$ & $-14.94 * *$ & $18.59 *$ & 18.82 & 0.51 & -3.58 & -19.12 \\
\hline 26 & IR-68888A x JGL 11118 & $-15.51 * *$ & $-9.60 * *$ & $-34.36 * *$ & -10.79 & $-30.71 * *$ & -11.26 & -7.80 & -3.58 & -2.57 & $-26.06^{*}$ \\
\hline 27 & IR-68888A x MTU 1010 & $-12.83 * *$ & $-9.20 * *$ & $-28.10 * *$ & -12.22 & $-23.24 * *$ & -4.56 & 7.54 & $-11.18^{*}$ & $-9.92 * *$ & $-35.83 * *$ \\
\hline 28 & IR-68888A x MTU1001 & $-9.09 * *$ & $-6.00 * *$ & $-30.65 * *$ & $-19.22 * *$ & $-27.80 * *$ & -2.97 & 11.10 & $-12.72 *$ & $-11.81 * *$ & $-38.53 * *$ \\
\hline 29 & IR-68888A x RNR 2458 & $-4.28 * *$ & -0.40 & $-11.98 * *$ & 10.97 & $-27.39 * *$ & $-42.99 * *$ & $-44.84 * *$ & 3.19 & $-21.20 * *$ & $-64.46^{* *}$ \\
\hline 30 & IR-68888A x PS 4 & $-9.09 * *$ & -6.40 & $-22.27 * *$ & $-17.16^{*}$ & -7.05 & $-21.15^{*}$ & $-24.16^{*}$ & 3.80 & 0.15 & $-39.68 * *$ \\
\hline 31 & IR-68888A x PS 5 & $3.74 * *$ & 4.40 & $-21.00 * *$ & $-23.41 * *$ & $-11.62 * *$ & $25.50 * *$ & $25.62 *$ & -0.02 & $-7.12 * *$ & -17.07 \\
\hline 32 & IR-68888A x BPT 5204 & $-8.02 * *$ & $-6.00 *$ & $-22.80 * *$ & $-21.76 * *$ & $-14.94 * *$ & 8.71 & 19.58 & -9.09 & $-28.85^{* *}$ & $-39.84 * *$ \\
\hline 33 & IR-68888A x Sumati & $2.14^{*}$ & 1.20 & $-27.15 * *$ & 7.00 & $-18.26^{* *}$ & $53.21 * *$ & $38.61 * *$ & $10.34 *$ & $-18.73 * *$ & $41.04 * *$ \\
\hline 34 & IR-68888A x WGL 32100 & -0.53 & -0.40 & $-36.06 * *$ & -6.06 & $-28.22 * *$ & $-31.03 * *$ & -18.57 & $-15.34 * *$ & $-13.89 * *$ & $-49.50 * *$ \\
\hline 35 & IR-68888A x WGL 283 & $2.67 *$ & $3.60 * *$ & $-29.69 * *$ & $-14.89 *$ & $-21.99 * *$ & $36.14 * *$ & $38.28 * *$ & -1.71 & $-12.78 * *$ & -5.54 \\
\hline 36 & IR-68888A x WGL 3962 & $-8.02 * *$ & $-6.00 * *$ & $-26.41 * *$ & -9.90 & $-22.41 * *$ & 0.21 & 9.47 & -8.60 & $-15.54 * *$ & $-33.16 * *$ \\
\hline 37 & IR-68897A x NLR 145 & -1.60 & -1.60 & $-27.89 * *$ & $-16.76^{*}$ & $-24.07 * *$ & $17.07 *$ & $23.26 *$ & -4.64 & $-18.64 * *$ & $-25.48 *$ \\
\hline 38 & IR-68897A x NLR 34449 & $-5.35 * *$ & $-3.60 * *$ & $-19.19 * *$ & $-13.73 *$ & $-10.79 * *$ & $33.24 * *$ & $30.08 * *$ & 2.40 & $-6.29 *$ & 5.59 \\
\hline 39 & IR-68897A x NLR 40024 & 0.00 & $2.40^{*}$ & $-20.25 * *$ & $-17.21 *$ & $-9.13^{*}$ & $26.74 * *$ & $37.76^{* *}$ & -8.11 & $-13.70 * *$ & -6.46 \\
\hline 40 & IR-68897A x NLR 3041 & 1.07 & 0.80 & $-22.69 * *$ & 2.59 & $-11.20 * *$ & $59.64 * *$ & $68.44 * *$ & -5.27 & $-14.23 * *$ & $50.54 * *$ \\
\hline 41 & IR-68897A x NLR 3010 & $3.74 *$ & $2.80^{*}$ & $-22.69 * *$ & $15.02 *$ & $-14.52 * *$ & $-60.61 * *$ & $-55.49 * *$ & $-11.69 *$ & $-23.18 * *$ & $-72.54 * *$ \\
\hline 42 & IR-68897A x NLR 3083 & $-2.14 *$ & $-2.40^{*}$ & $-18.88 * *$ & -9.99 & -7.05 & $47.13 * *$ & $43.13 * *$ & 3.24 & $-6.24 *$ & $32.43 * *$ \\
\hline
\end{tabular}


Int.J.Curr.Microbiol.App.Sci (2018) 7(9): 2018-2025

\begin{tabular}{|c|c|c|c|c|c|c|c|c|c|c|c|}
\hline $\begin{array}{l}\text { S. } \\
\text { No }\end{array}$ & Cross & $\begin{array}{l}\text { Days to } \\
50 \\
\text { flowering }\end{array}$ & $\begin{array}{l}\text { Days to } \\
\text { maturity }\end{array}$ & $\begin{array}{l}\text { Plant } \\
\text { height } \\
\text { (cm) }\end{array}$ & $\begin{array}{c}\text { No. of } \\
\text { productive } \\
\text { tillers }^{-1} \\
\text { plant }^{-1}\end{array}$ & $\begin{array}{l}\text { Panicle } \\
\text { length } \\
(\mathrm{cm})\end{array}$ & $\begin{array}{c}\text { No. of } \\
\text { filled } \\
\text { grains } \\
\text { panicle- }{ }^{1}\end{array}$ & $\begin{array}{l}\text { Total } \\
\text { no. of } \\
\text { grains } \\
\text { panicle-1 }\end{array}$ & $\begin{array}{l}\text { Spikelet } \\
\text { fertility } \\
\text { (g) }\end{array}$ & $\begin{array}{c}\text { Test } \\
\text { weight } \\
\text { (g) }\end{array}$ & $\begin{array}{l}\text { Grain } \\
\text { yield } \\
\text { plant-1 } \\
\text { (g) }\end{array}$ \\
\hline 43 & IR-68897A x ADT 37 & $5.35 * *$ & $4.00 * *$ & $-21.63 * *$ & $-33.75 * *$ & $-13.28 * *$ & $-34.69 * *$ & -14.14 & $-23.31 * *$ & 0.68 & -72.22 \\
\hline 44 & IR-68897A x JGL 11118 & $-4.81 * *$ & $-3.60 * *$ & $-18.88 * *$ & $-18.32 * *$ & $-11.20 * *$ & $46.99 * *$ & $46.00 * *$ & 1.13 & -3.97 & 19.50 \\
\hline 45 & IR-68897A x MTU 1010 & 1.07 & 1.60 & $-22.69 * *$ & -11.41 & $-11.20 * *$ & 8.71 & $23.73^{*}$ & $-12.08 *$ & $-11.57 * *$ & -19.39 \\
\hline 46 & IR-68897A x MTU1001 & -1.60 & 0.00 & $-13.04 * *$ & $-13.69 *$ & $-8.71 *$ & $59.92 * *$ & $51.43 * *$ & 5.84 & -1.21 & $43.44 * *$ \\
\hline 47 & IR-68897A x RNR 2458 & 1.60 & 0.00 & $-18.45^{* *}$ & $-27.69 * *$ & $-10.79 * *$ & $49.97 * *$ & $52.07 * *$ & -1.54 & $-17.91 * *$ & -17.05 \\
\hline 48 & IR-68897A x PS 4 & 1.60 & 1.20 & $-24.50 * *$ & $-17.70 *$ & -4.98 & $28.33^{* *}$ & $28.87 * *$ & -0.54 & $-13.26^{* *}$ & -12.24 \\
\hline 49 & IR-68897A x PS 5 & $-3.74 * *$ & -1.20 & $-23.65^{* *}$ & -12.30 & $-10.37 *$ & -2.83 & 9.59 & $-10.63 *$ & $-12.10 * *$ & $-32.61 * *$ \\
\hline 50 & IR-68897A x BPT 5204 & $-8.02 * *$ & $-4.40 * *$ & $-15.59 * *$ & $-23.41 * *$ & -6.64 & $53.49 * *$ & $60.08 * *$ & -4.23 & $8.95 * *$ & $32.98 * *$ \\
\hline$\overline{51}$ & IR-68897A x Sumati & $3.21 * *$ & $2.40 *$ & $-22.80 * *$ & 0.76 & $-14.52 *$ & $-63.51 * *$ & $-58.04 * *$ & $-13.22 * *$ & $-11.91 * *$ & $-74.60 * *$ \\
\hline 52 & IR-68897A x WGL 32100 & -1.07 & -0.40 & $-19.19 * *$ & 0.94 & -4.98 & $51.14 * *$ & $38.68 * *$ & 8.89 & $-19.36 * *$ & $29.60 * *$ \\
\hline 53 & IR-68897A x WGL 283 & $-6.42 * *$ & $-4.40 * *$ & $-19.83 * *$ & -6.24 & -5.81 & $43.95 * *$ & $39.74 * *$ & 2.91 & $-6.63 * *$ & $32.85^{* *}$ \\
\hline 54 & IR-68897A x WGL 3962 & $-2.14 *$ & -0.40 & $-15.16^{* *}$ & $-23.85^{* *}$ & $-12.03 * *$ & 9.88 & 20.34 & -8.87 & $-15.10 * *$ & $-28.24 *$ \\
\hline
\end{tabular}


With regard to test weight, standard heterosis ranged from -33.11 (IR-58025A x NLR 3041) to 11.28 (IR-58025A x WGL 3962) and two hybrids were recorded significant positive heterosis over KRH-2.

\section{Grain yield plant $^{-1}(\mathrm{~g})$}

Grain yield per se is a complex heritable character which is an end product of multiplicative interaction of various yield components and hence heterosis for yield may be attributed to heterosis of individual yield components or alternatively due to multiplicative effects of component characters. The present investigation revealed that, a wide range of variation in the estimates of standard heterosis in positive and negative direction was observed for grain yield per plant. Heterosis for grain yield is mainly because of simultaneous manifestation of heterosis for other yield components. The desirable positive significant heterosis for trait grain yield per plant this was exhibited by ten hybrids with a range of -74.60 (IR-68897A x Sumati) to 50.54 (IR-68897A x NLR 3041) over check KRH-2.

The hybrid IR-68897Ax NLR 3041 (50.54) recorded the highest standard heterosis for grain yield per plant followed by IR-68897A x MTU1001 (43.44), IR-68888A x Sumati (41.04), IR-58025A x WGL 3962 (33.48), IR68897A x BPT 5204 (32.98), IR-68897A x WGL 283 (32.85), IR-68897A x NLR 3083 (32.43), IR-68897A x WGL 32100 (29.60), IR-58025A x NLR 34449 (26.08) and IR58025A x NLR 3083 (22.59) over check.

Several authors Chouhan et al., (2016), Nainu et al., (2016), Borah et al., (2017), Dharwal and Verma (2017) and Parameshwar Kumar et al., (2017) reported both positive and negative heterosis in their studies for all these traits. For grain yield per plant, negative to positive heterosis over standard check reported by Tiwari et al., (2011), Adilakshmi et al., (2013), Venkatasubbaiah et al., (2013), Devi et al., (2014), Chouhan et al., (2016), Nainu et al., (2016), Borah et al., (2017), Dharwal and Verma (2017) and Parameshwar Kumar et al., (2017).

Higher magnitude of heterosis in predominant number of crosses over standard check was observed for number of filled grains per panicle, total number of grains per panicle and grain yield per plant. In present investigation, hybrids showing significant positive standard heterosis could be most promising hybrids, suitable for heterosis breeding and need to be tested in large scale so that they may be considered for commercial exploitation.

\section{References}

Adilakshmi, D., Satyanarayana, P.V., and Vijayalakshmi, B. 2013. Studies on heterosis and combining ability of bacterial leaf blight donors in rice (Oryza sativa L.). The Andhra Agricultural Journal. 60 (2): 320-325.

Borah, P., Sarma, D., and Hazarika, G.N. 2017. Magnitude of heterosis for yield and its components in hybrid rice (Oryza sativa L.). International Journal of Agricultural Science and Research. 7 (2): 209-216.

Central Rice Research Institute-Vision 2050, Indian Council of Agricultural Research, Cuttack, Odisha-753 006. PP No.8.

Chouhan, S. K., Agrawal, R.K., Singh, R.P., and Pratapa Reddy, M. 2016. Heterotic expression of rice (Oryza sativa L.) hybrids for yield and some yield components. International Journal of Applied Biology and Pharmaceutical Technology. 7 (4): 86-93.

Devi, R, K., Parimala, K., and Cheralu, C. 2014. Heterosis for yield and quality 
traits in rice (Oryza sativa L.). The Journal of Research, ANGRAU. 42(1): $1-11$.

Dharwal, G., and Verma, O. P. 2017. Exploitation of heterosis in yield and its components in rice. International Journal of Applied Bioscience. 5 (2): $77-80$

Liang, G.H., Reddy, C.R., and Dayton, A.D. 1971. Heterosis, inbreeding depression and heritability estimates in a systematic series of grain sorghum genotypes. Crop Science. 12: 409-411.

Nainu, A.J., Palaniraja, K., and Vennila, S. 2016. Effect of coastal saline environment on heterosis in hybrid rice. Life Science Archives (LSA), 2 (3): 565569.
Parameshwar Kumar, S., Deepak, S., Singh, S., Kumar, K.I., and Chand, S.F. 2017. Heterotic expressions evaluation for grain yield and quality traits in CMS based rice hybrids. International Journal of Genetics. 9(3). 257-262.

Tiwari, D.K., Pandey, P., Giri, S.P., and Dwivedi, J.L. 2011. Heterosis studies for yield and its components in rice hybrids using CMS system. Asian Journal of Plant Sciences. 10 (1): 2942.

Venkatasubbaiah, P., Sekhar, M.R., Reddy, K. H. P., and Eswarreddy, N. P. 2013. Heterosis for yield and yield components in CMS based hybrids in rice (Oryza sativa L.). The Andhra Agriculture Journal. 60(2): 292-296.

\section{How to cite this article:}

Krishna Naik, R., P. Ramesh Babu, J. Dayal Prasad Babu, Y. Ashoka Rani and Srinivasa Rao, V. 2018. Exploitation of Heterosis for Yield and Its Components in Rice. Int.J.Curr.Microbiol.App.Sci. 7(09): 2018-2025. doi: https://doi.org/10.20546/ijcmas.2018.709.244 